\title{
Molecular Mechanisms for Drosophila Neuronetwork Formation
}

\author{
Marie-Pierre Furrer Akira Chiba \\ Department of Cell and Structural Biology, University of Illinois, Urbana, III., USA
}

\author{
Key Words \\ Drosophila · Axon · Dendrites · Guidance · Branching • \\ Targeting
}

\begin{abstract}
Understanding the establishment of functional neuronetworks is one of the frontiers of developmental neurobiology. The use of axons and dendrites from Drosophila nervous system as a model allows the identification of molecular mechanisms which give neurons the ability to guide their processes en route to connect precisely with their partners. By focusing on selected Drosophila model systems, we discuss the recent advances in our understanding of the molecular mechanisms regulating guidance, branching and targeting of axons and dendrites required for the establishment of a functional neuronetwork.
\end{abstract}

Copyright @ 2004 S. Karger AG, Basel

\section{Introduction}

One of the essential requirements for constructing a functional neuronetwork is correct matchmaking between pre- and post-synaptic partners. To achieve this, a neuron seeks its partners while extending multiple processes, typ- ically a single axon and multiple dendrites, often over a rather long distance. This correct matchmaking requires that the neuron follows a succession of steps such as growth, guidance and branching of processes as well as their correct targeting and synaptogenesis. Understanding the construction of a functional neuronetwork implies understanding the molecular mechanisms of axonal and dendritic growth, pathfinding and target recognition.

Drosophila has been used for decades to elucidate various developmental processes through its powerful genetic tools. The analyses of mutants and the possibility to conduct genetic screening for particular phenotypes have been applied to decipher the molecular mechanisms leading to a functional neuronetwork. The constant development of genetic and cell visualization tools has allowed Drosophila neurobiologists to generalize the use of various neuronal models at different developmental stages. It also allowed the conduction of analyses at the single-cell level, as well as single-cell restricted genetic manipulations. Several neuronal models have been chosen for their best adequacy to study a particular step in the formation of functional neuronetwork. The study of neuronetwork development in such models highlights some constants in the programming of neuron connectivity. In this review, we discuss the molecular mechanisms controlling several important steps a neuron has to take to meet its correct partners and start long-term relationships with them.

\begin{tabular}{ll}
\hline KARGER & (c) 2004 S. Karger AG, Basel \\
$\begin{array}{l}\text { Fax + 41 61 306 1234 } \\
\text { E-Mail karger@karger.ch } \\
\text { www.karger.com }\end{array}$ & $\begin{array}{l}\text { Accessible online at: } \\
\text { www.karger.com/nsg }\end{array}$
\end{tabular}

Marie-Pierre Furrer

B605 Chemical and Life Sciences Laboratories

601 South Goodwin Avenue

Urbana, IL 61801 (USA)

Tel. +1 217244 8963, Fax +1 217244 8962, E-Mail mfurrer@uiuc.edu 


\section{Guidance}

\section{Checkpoint Decision at the CNS Midline}

Once a neuron is born, it starts to grow processes that will become the axon and dendrites. The mechanisms that regulate the polarity of the neuron as well as where the axon and dendrites grow are yet to be understood, but it appears to be a controlled process. To grow over a long distance, axons rely on the interpretation of both longrange and short-range cues provided by the environment. They follow trajectories that are staked out with multiple checkpoints, dividing axon guidance into a series of decision-making events [1].

The CNS of bilateral animals is physically divided by the midline. To ensure coordination between the two sides of the brain, communication has to be established and also regulated. To this end, specific neurons send their processes, either axon or dendrites, across the midline, creating communication lines displayed as commissures. Therefore, CNS neurons have two choices regarding their projections, remaining ipsilateral and avoiding the midline or projecting contralaterally and going through the midline. The midline, with its yes or no question, is a perfect paradigm for studying how neurons make a directionality choice at a checkpoint.

In Drosophila, the embryonic abdominal CNS is constituted by a repetition of identical segments (segments A2-A7), each of which is composed of mirror-image hemisegments. Each abdominal hemisegment is reported to contain 342 neurons [2], including 34 that are motoneurons exiting the CNS to innervate 30 abdominal muscles $[2,3]$. The position of neurons, as well as the directionality of their projections, both axons and dendrites, and their targets are highly stereotypic, conserved from one hemisegment to another and from one animal to another, making the ventral nerve cord an ideal model for genetic and cellular analysis. The midline is materialized by the presence of three essential glial cells, hereafter referred to as midline glia cells per segment. Several markers expressed on neuron projections allow the visualization of the wild-type stereotypic organization of the embryonic ventral nerve cord. Among them, the antibody BP102 labels specifically the whole embryonic neuropile revealing a 'ladder-like' structure with longitudinal connectives connected by a pair of anterior and posterior commissures in each segment [4]. As for the question of midline, crossing the embryonic nervous system can be simplified as a model in two dimensions with ipsilateral and contralateral neuropiles linked by commissures.
BP102 antibody has been used widely to identify mutations disrupting the general organization of the neuropile. These mutations can be classified into two main groups. The first group includes mutations for which the commissures appear thickened by an excess of fibers crossing the midline. The second group consists of mutations for which the commissures look thinner due to the reduction in the number of fibers crossing the midline. These opposite phenotypes suggested a model in which excess crossing is due to the lack of activation of a pathway triggering repulsion, and reduced crossing is due to the absence of activation of an attraction pathway [1]. Several molecules that generate this disorganization of midline crossing have been identified and their function characterized. They exemplify how neurons rely on their extracellular environment for their guidance, while emphasizing the importance of midline glia cells as an organizing center of the CNS projections [5].

Midline glia cells secrete ligands that activate receptors localized on the surface of neurons, triggering either attraction or repulsion. Neuronal response depends on the receptor they activate, leading the neuronal processes to cross the midline or not.

NetrinA and NetrinB, secreted by midline glia, interact with their receptors, Frazzled and Unc5 [6-9]. When frazzled is mutated, commissures appear greatly reduced and the longitudinal tracts are periodically interrupted [7]. Conversely, when Unc5 is overexpressed in all neurons, processes fail to cross the midline [9]. Both responses are dependent on Netrins, but neither receptor requires the other for its function [9]. Therefore, it appears that Netrins are bifunctional molecules capable of driving attraction through interaction with Frazzled or repulsion through interaction with Unc5.

Midline glia cells also secrete Slit, the ligand for the Roundabout family of transmembrane receptors, Roundabout, Robo2 and Robo3 [4, 10-13]. When Slit function is disrupted, a collapse of the entire neuropile to the midline is observed $[10,11]$. When roundabout is mutated, an excess of fibers crossing the midline is observed [4]. This suggests that the activation of Roundabout by Slit triggers repulsion of neuronal processes away from the midline. Although both Unc5/Netrins and Roundabout/slit drive repulsion by the midline, it seems that they operate independently from each other [9].

A study using mammalian cell culture suggests that the repulsion driven by roundabout signaling is due to an association of activated Roundabout with N-cadherin, a mediator of homophilic cell adhesion. This association disrupts the link between $\mathrm{N}$-cadherin and the actin cyto- 
skeleton, disabling cadherin-mediated adhesion [14]. This suggests that a growth cone expressing Roundabout at its surface and in the vicinity of the midline has its Roundabout receptors activated, which leads to disruption of adhesion. Thus, the response to attraction/repulsion signaling would be by sticking/sliding.

Analysis of the expression pattern suggests that most if not all neurons express roundabout [4], suggesting a regulatory mechanism that allows some neurons to send their processes across the midline. Immunodetection of Roundabout showed that the protein is nearly excluded from the commissures [15]. Commissureless, whose mutation makes the neuronal processes unable to cross the midline, is cell-autonomously responsible for the downregulation of Roundabout in commissural axons [16]. Commissureless downregulates Roundabout by diverting it to the endosome pathway, where it is thought to be degraded [16], hindering its activation by Slit. Commissureless is itself regulated by an ubiquitin ligase DNedd 4 and an interaction between Commissureless and DNedd 4 is required for downregulation of Roundabout [17]. The expression of Commissureless is believed to be restricted to the neurons sending their axon across the midline [16, 18]. It also appears to be highly dynamic [16], suggesting that the downregulation of Roundabout has to occur with a precise timing during the course of axon guidance.

With its stereotypical organization, the ventral nerve cord of Drosophila allows identified single-cell analysis of various mutants. These analyses and single-cell genetic manipulations can be achieved with the use of the Gal4/ UAS system [19]. Gal4 drives transgene expression that can be restricted to a subset of cells per hemisegment. Alternatively, fluorescent dyes can be applied directly to the cell of interest, allowing the visualization of the morphology of an entire identified neuron [3, 20,21]. Such analyses have shown that axon and dendrites of an unidentified neuron can respond differentially to mutations of roundabout or frazzled. Among the motoneurons studied, the aCC motoneuron (anterior Corner Cell) possesses an axon that remains ipsilateral and two populations of dendrites, one that crosses the midline and the other one that remains ipsilateral. In frazzled mutant embryos, aCC's axonal projection remains unaffected whereas the crossing dendrites are missing, suggesting a requirement of Frazzled for dendritic crossing. In roundabout mutant embryos, both axonal and dendritic projections remain as described for wild-type. The RP3 motoneuron, also studied at the single-cell level, projects its axon across the midline to innervate contralateral muscles. RP3 possesses two populations of dendrites, growing away on each side of the midline. In frazzled mutant embryos, RP3's axon fails to cross the midline, whereas in roundabout mutant embryos, dendrites fail to escape the midline, while the axon remains unaffected and targets its contralateral muscles as in wild-type [22,23]. These data, as well as the genetic manipulations resupplying the wildtype product of the mutant gene to a small subset of cells show that one process can be cell-autonomously, or more precisely process-autonomously, attracted towards the midline, whereas the other is diverted from it [22, 23]. Such data show that in addition to cell-type-specific regulation of midline signaling through the regulation of Roundabout function, neurons are subcellularly capable of integrating differentially opposite signals in axon and dendrites. The ability to visualize and genetically manipulate a single identified cell makes Drosophila an ideal in vivo model to dissect the subcellular regulations involved in the response to midline signaling.

Studies in different vertebrate models have suggested a model by which cells that express both frazzled and roundabout $[4,7]$ are able to drive their processes towards or away from the midline. In this model, the activation of Roundabout by Slit unlashes the binding of its cytoplasmic domain with the one of DCC/Frazzled, thus silencing the attraction signals [24]. Single-cell analyses have shown that processes originating from same cell respond to both Roundabout and Frazzled signaling independently [23]. This suggests that the silencing of the attraction signals by Roundabout and the downregulation of repulsion signaling by Commissureless are strictly restricted in terms of localization and timing, conferring processes such independence of response.

\section{CNS Fascicles}

In addition to facing the question of whether to remain ipsilateral or to cross the midline, each embryonic neuron selects and fasciculates with one of some 20 longitudinal fascicles that are positioned on either side of the midline. Specific markers expressed on these different fascicles characterize them, as exemplified by the use of anti-Fasciclin antibody (FasII). FasII antibody labels three major parallel pathways on each side of the midline [25]. Each of these three pathways is composed of several fascicles with similar mediolateral positioning but different dorsoventral positions [25-27]. This pattern of expression suggested that cell adhesion molecules are essential for the selection of a longitudinal pathway. How do neurons differentiate between several similar pathways? The characterization of Slit receptors, Roundabout, Robo2 and Robo3, gives insights as to how similar lateral pathways are dis- 
criminated $[12,13]$. Roundabout is expressed in the whole CNS, whereas Robo3 expression is limited to the medial and lateral regions, and Robo 2 expression is restricted to the most lateral part of the CNS $[12,13,28]$. In addition, all three Robo receptors are absent from the commissures, due to their downregulation by Commissureless [12, 28, 29]. By their combinatorial patterns of expression, these Robo receptors define three regions on each side of the midline, with Robo expression defining the most medial region, combination of Robo and Robo3 defining the intermediate region, and the addition of all three Robos defining the most lateral region of the neuropile $[12,13$, 28].

Analyses of loss of function of each of the three Robo receptors, either as a single mutant or as double mutants, as well as overexpression experiments, have shown the following. Roundabout is required primarily to control the crossing of the midline and to confine the longitudinal pathways on each side of the midline [15]. Robo2, on the other hand, contributes to the reading of the repulsion signal, showing that Robo and Robo2 share some function in control of midline crossing [12, 28, 29]. Robo2, however, also possesses its own function in determining which axons will join the most lateral pathways, whereas Robo3 controls the formation of the medial neuropile $[12,28$, 29]. These distinct functions are thought to be mediated by both the differences in the protein sequences and the spatiotemporal patterns of expression. Thus, it is proposed that the three Robo receptors establish a Robo code that addresses each axon in one of these regions where local cues and specific adhesion molecules determine the choice of a longitudinal pathway.

Outside the CNS, the neurons in the PNS of Drosophila embryos and larvae also offer a wonderful model. Each of the PNS sensory neurons have been identified and their stereotypical axonal projections have been characterized $[27,30]$. The position of sensory neurons' terminal projections can be mapped in respect to uniquely identified FasII fascicles $[25,26]$. Roundabout is found expressed in chordotonal neurons, which project their terminal arbor to an intermediate fascicle, and in multidendritic (md) neurons, which project onto the medial fascicle. Robo3 is detected only in chordotonal neurons, whereas Robo2 is not expressed in these types of sensory neurons [27]. Cells depleted of Roundabout, either by loss of function or downregulation through overexpression of Commissureless, send their terminal projections across the midline and form bilateral projections at the appropriate mediolateral and dorsoventral location, suggesting a similar role of Roundabout in confining projections on the ipsilateral side of the midline for PNS sensory neurons as described for CNS neurons [4, 15, 27]. Robo3, whose specific expression in chordotonal neurons is directed by Atonal, is required cell-autonomously for the positioning of chordotonal terminal arbors to the intermediate fascicle, as shown by cell-specific rescue experiments [27]. Misexpression of Robo3 in the dorsal bipolar dendritic (dbd) neuron, one of the md neurons, is sufficient to shift $\mathrm{dbd}$ terminal arbor from the medial to the lateral fascicle, showing that Robo3 is necessary and sufficient to control targeting to the intermediate fascicle [27]. As noted previously for CNS neurons, the dorsoventral positioning is not affected by manipulations of Roundabout or Robo3, suggesting its control by another set of signaling molecules $[12,27,28]$. The dorsoventral positioning might be directed by local cues, once the Robo code addresses CNS axons or PNS sensory terminals to its appropriate position along the mediolateral axis. Again, Slit appears to be the ligand of Roundabout and Robo3 for their function in the positioning of PNS terminal arbors. In addition, Slit also appears to function in the branching of terminals, consistent with the Slit role in dendritic branching observed in vertebrates [27, 31].

\section{Branching}

Besides correct pathfinding, the generation of a functional neuronetwork supposes that each constituent of the network will develop the correct shape. Having the right shape, or the right projections, is essential to contacting the correct partners. More importantly, recent studies have shown that the branching pattern as well as the protein composition of both axonal and dendritic arbors play a fundamental role in the electrical properties of a neuron as well as its ability to compute and store information [32, 33]. Thus, identifying the mechanisms that control branching is essential to the comprehension of the development of functional network.

The stereotypical organization of the Drosophila CNS as well as the fairly invariant pattern of branching that has been observed in several neuronal types illustrate the presence of genetic programming in axon and dendrite branching. Several different neuronal models have been selected to study either axon or dendrite branching, and have allowed the characterization of molecular mechanisms involved in the development of cell arbors.

The models used for the analysis of both axon and dendrite branching are typically analyzed in late developmental stages, either third instar larvae, pupae or adults.
40

Neurosignals 2004;13:37-49
Furrer/Chiba 
A genetic screen for branching mechanisms requires that the mutation studied or newly generated will not produce pleiotropic phenotypes or death at earlier developmental stages. The development of genetic tools allowing the generation of homozygous mutant clones in an heterozygous animal [34], complemented by the labeling of the mutant clones with Gal4/UAS system [19] and green fluorescent protein via the segregation of the repressor gene Gal80 (MARCM technique [35]), has overcome the problem of analyzing mutants at late developmental stages. The MARCM technique thus allows the generation of homozygous mutant clones in an heterozygous animal, the cells of the mutant clone being the only cells that express green fluorescent protein. The size of the clone can be controlled, allowing the visualization of single-cell or multiple-cell clones.

\section{Axon Branching}

The ability of a neuron to innervate several targets in different locations often depends on the formation of axonal branches. Two mechanisms can lead to the formation of such branches. Sister branches can either develop after the first branch extends, as a collateral extending from a preexisting branch, or they can develop simultaneously, through a splitting growth cone, which gives two sister branches [36, 37].

Mushroom bodies (MBs), a brain structure conserved in Arthropods, appear as a paired bilateral structure. Most axons in MBs possess two major processes that project away from each other at a right angle into distinct target fields [38-40]. MBs play an essential role as they are centers for higher-order functions, including olfactory learning and memory [41, 42].

MBs are derived from four neuroblasts in each hemisphere, each of which produces all three major types of neurons. These neurons are distinguished by their morphological characteristics and their birth order during development $[43,44]$. These three types of neurons are $\gamma$, $\alpha^{\prime} / \beta^{\prime}$ and $\alpha / \beta$ neurons, which project into morphologically distinct lobes. Cell bodies of MB neurons, called Kenyon cells, are packed in the dorsal cortex and extend their dendrites anteriorly into the calyx, which receives olfactory information from the antennal lobes. Distally, MB axons fasciculate into a bundle called peduncle, which splits at its most anterior position to form medial $\left(\gamma, \beta^{\prime}\right.$ and $\left.\beta\right)$ and dorsal $\left(\alpha^{\prime}, \alpha\right)$ lobes [45].

The four MB neuroblasts, born at an early embryonic stage [46], proliferate continuously throughout development $[47,48]$. Clonal analysis demonstrated that each single MB neuroblast produces all 3 types of MB neurons, with generation of $\gamma$ neurons first, then the $\alpha^{\prime} / \beta^{\prime}$ neurons, and finally $\alpha / \beta$ neurons [44]. As proliferation continues, the MB axons are organized into layers, with the projections from the youngest neurons in the center and progressively shifted more laterally, as new axons grow into the peduncle [49]. At first, all these neuron types present branching into medial and dorsal lobes, but during metamorphosis, $\gamma$ lobes are remodeled through selective pruning and retain only their medial lobes [44, 50]. Thus, MB neurons are capable of producing axon branches that target different regions of the brain.

How is axon branching regulated? A screen using the MARCM technique has been conducted and led to the identification of Dscam (Down syndrome cell adhesion molecule) as a regulator of axon branching. In Dscam mutant MBs, the $\gamma$ lobes appear normal, whereas the $\alpha / \beta$ lobes appear much thicker and denser than wild-type MBs. In addition, mutant clones project their bifurcating axons in a single lobe [51]. Further analyses have shown that Dscam is required for axon bifurcation and guidance of both $\alpha^{\prime} / \beta^{\prime}$ and $\alpha / \beta$ axons through cell-autonomous and non-cell-autonomous effects [51]. Single cell mutant clone analysis revealed that $\alpha^{\prime} / \beta^{\prime}$ and $\alpha / \beta$ neurons lacking Dscam present supernumerary branches deriving from repeated bifurcation. These mutant neurons also failed to segregate branches into the two lobes, showing a cellautonomous requirement of Dscam for the control of branch number and the proper segregation of sister branches [51]. Since Dscam is expressed in the nervous system throughout development and there is a similar role of Dscam in another brain structure [51], it is possible that Dscam could be used throughout development to control branching. With its 38,016 possible alternative splice forms [52] however, Dscam might possess numerous functions besides regulation of branching, as underlined by mistargeting of Dscam mutant olfactory neurons to the antennal lobe glomeruli, which shows a function of Dscam in targeting specificity [53].

The branching of MB axons to form medial and lateral lobes by bifurcation suggests that axon growth, guidance and branching occur more or less simultaneously. Increasing defects in MB axon branching, guidance and growth were observed with the progressive removal of wild-type copies of the three Rac GTPases Rac1, Rac2 and Mtl, with axonal branching being the most sensitive to loss of Rac activity [54]. This suggests that, while occurring concomitantly, growth, guidance and branching are distinct events requiring different levels of activation for the Rac proteins. It also appears that these three events are generated through different effector pathways, since CRIB- 
domain effectors of the GTPases, like Pak, are proposed to contribute only to guidance and branching, but not to axon growth [54]. These studies on the contribution of Rho GTPases activity shed some light on how a neuron manages to control different events all involving cytoskeleton rearrangements.

\section{Remodeling Axonal Branches}

Holometabolous insects like Drosophila undergo a complete metamorphosis between larval and adult stages. Three fates await larval neurons during metamorphosis. They can remain unchanged, they can be removed by apoptosis, or they can go through a phase of remodeling by selective pruning of axons and dendrites, allowing the reintegration of these neurons in adult neuronetworks $[55,56]$. This removal of specific neuronal processes in a temporarily controlled manner implies the existence of a genetic program for pruning.

In MBs, while $\alpha^{\prime} / \beta^{\prime}$ and $\alpha / \beta$ lobes remain unchanged during metamorphosis, $\gamma$ neurons specifically retract their dorsal and medial branches and re-extend into a single medial lobe $[44,50]$. This offers a model for the study of neuronal remodeling. The pruning of the $\gamma$ lobe starts with the disruption of the microtubule cytoskeleton and continues with the appearance of blebbing in dendrites and then in axons, followed by fragmentation and removal of both processes. This suggests that pruning occurs by local degeneration rather than retraction [50].

The similarities in phenotypes where the $\gamma$ neurons fail to remodel during metamorphosis are observed in Uba1 (ubiquitin-activating enzyme-1), as well as in Mov34 and Rpn6 (two subunits of the 19S proteasome-regulatory particle required for polyubiquitinated protein degradation) mutant MB clones, suggesting that proteasome-mediated protein degradation after polyubiquitination is essential for axon pruning [50]. Additional phenotypes observed in these mutant clones suggest pleiotropic functions of protein ubiquitination in different phases of neuronal development [50].

Metamorphosis is under the control of the steroid molting hormone 20-hydroxyecdysone. EcR-B1, one of the isoforms of ecdysone receptor, is specifically involved in the remodeling of larval cells $[57,58]$. This EcR isoform is expressed in $\gamma$ neurons and required for their pruning, and absent in the other MB neurons [59]. In these $\gamma$ neurons, the activation of Baboon (a TGF- $\beta$ /activin type-I receptor [60]) by dAct, a member of the activin subfamily [61], induces its heterodimerization with either Wishful thinking or Punt (two constitutively active TGF$\beta$ /activin type-II receptors [62-64]), which can act redun- dantly in $\gamma$ neurons [65]. dSmad2, baboon's downstream transcriptional effector $[60,66]$, transduces the TGF- $\beta$ signal to the nucleus where it directly or indirectly controls the expression of EcR-B1 [65]. Thus, the forced expression of EcR-B1 in baboon mutant $\gamma$ neurons specifically restores remodeling during metamorphosis [65]. The ubiquitin proteasome system has no function in the control of EcR-B1 expression in $\gamma$ neurons [50]. It is not known if $\gamma$ neuron pruning requires other cell-type-specific proteins or if EcR-B1 expression is sufficient to induce metamorphosis-related remodeling.

The study of axon branching puts emphasis on the role of downstream signaling pathways with the involvement of Rho GTPases. It appears that these molecules act in different steps of neuronal differentiation, including axon guidance. Furthermore, the fact that different levels of activation of Rho GTPases could control these different steps sheds some light on how neurons reuse similar pathways for different purposes. Although, in MBs, branching and growth occur at the same time, in other neuronal systems such a coupling is less evident or there might be several other decision events coupled with growth. In these cases, additional factors might be required for successfully reusing similar signaling pathways. It is thus possible that timing of events, as well as the use of different sets of effectors or the strict control of downstream pathway protein localization and activation permit neurons to use same pathways to different events.

\section{Dendritic Branching}

Each abdominal hemisegment of Drosophila embryos and larvae contains 44 peripheral neurons that have been mapped into dorsal, lateral and ventral clusters [67]. Their position as well as their targets and their cellular shape (dendritic arbor and cell size) led to the identification of three neuronal types: external sensory (es) neurons, chordotonal (ch) neurons (both with single, unbranched dendrites) and md neurons [68-70]. Of those, the dendritic arborization (da) neurons present the most developed dendritic arbor. They have been further characterized and chosen as a privileged model for dendritic growth and branching.

The growth of da dendrites starts $2 \mathrm{~h}$ after the axons of PNS have reached the CNS, with the emergence at an invariant location of a primary dendritic branch which grows toward the dorsal midline [71, 72]. The growth of the dorsal primary branch continues for several hours, before being replaced by the extension of numerous transient lateral branches along the anteroposterior axis. Some of these lateral branches are stabilized before the 
growth of tertiary branches. Altogether, these successive, stereotypic steps of dendritic growth form the complex, reproducible pattern of dendritic arbor observed in da neurons [72]. Furthermore, single-cell analysis revealed that da neurons develop four different types of dendritic arborizations (class I to class IV) with increased levels of complexity and the presence of spiked-like structures for the class-III dendrites [73].

This morphological classification of dendritic complexity has been reported to be coincident with the level of expression of the homeodomain transcription factor cut. Class-I neurons display no Cut immunoreactivity, while low levels of Cut in class II, medium in class IV and high in class III are observed [74, 75]. Cut is primarily known for its role in the specification of cell identity [71]. However, cut expression persists in post-mitotic cells [74, 76]. Single-cell analysis of $c u t$ function in post-mitotic da neurons shows a crucial role of $c u t$ in the acquisition of classspecific morphology, and modulation of its expression is sufficient to change the morphology of da dendrites [75]. As for Cut, a putative transcription factor called Hamlet controls both cell fate specification and dendritic morphology [77]. In hamlet mutants, es neurons are transformed in md neurons with full $\mathrm{md}$ arbor morphology and post-mitotic expression of ham in md neurons transform their dendritic arbor but not their md fate [77]. Although these two transcription factors seem to control both neuronal cell fate and dendritic arbor specification, they appear to do so differently. Hamlet appears to be acting as a switch between two types of dendrite morphology, thus promoting, by its expression, single-dendrite morphology and repressing multiple arbors. In contrast, it is the level of Cut, rather than its expression itself, that selects the level of complexity of da neuron arbor. In addition, Hamlet controls a type of dendritic arbor in relation with a cell-type, whereas Cut controls different levels of dendritic complexity within a same cell-type.

Once the type of dendritic morphology has been determined by transcription factors, unidentified downstream programs are activated to comply. Mutation of some of the genes involved causes overextension of the dorsal branch that grows towards the dorsal midline. In sequoia mutants, the dorsal branch of md neurons starts its growth earlier and overextends to cross the dorsal midline and intermingles with the contralateral md neurons [72, 78]. Sequoia encodes a putative transcription factor that apparently controls cell-fate specification and dendritic morphology independently [78]. The identification of transcription factors with dual roles, controlling both celltype specification and specific branching pattern, suggests that neurons rely on intrinsic programming to extend their dendritic field.

As for sequoia, flamingo, a G-coupled transmembrane protein with extracellular cadherin domains [79], controls the size of the dendritic field. When flamingo is mutated, overextension of the dorsal branches of md neurons is observed [72, 80, 81]. Flamingo does not control the dendritic branching pattern in a global way; it only limits the extension of the dorsal branches by controlling the timing of dendritic extension [80, 81]. Since Flamingo can work as a homophilic cell-adhesion molecule in vitro, it is suggested that its control of the timing of dendritic growth and of the dendritic field goes through homophilic interactions. The involvement of both sequoia and flamingo in the control of the size of dendritic field shows that the branching pattern of da neurons is under the control of both extrinsic and intrinsic factors. The requirement of Flamingo to control the size of dendritic field is not limited to PNS sensory neurons, since flamingo mutant MBs also present overextended dendrites [82]. How might the transmembrane signal translate into dendritic extension? Loss of function of tropomyosin II has been shown to result in overgrown dendritic fields of da neurons [83]. Tropomyosin II stabilizes actin filaments and increases their strength [84]. The genetic interaction of tropomyosin II with flamingo provides a potential link between flamin$g o$ and the actin cytoskeleton. Thus, Flamingo signaling leads to a stabilization of actin filaments and therefore controls the size of dorsal branches [83]. Laser ablation experiments and the use of specific mutants reducing the number of PNS neurons generated suggests that the dendritic field of homologous neurons are shaped by competition between neighboring cells, since they repel each other at the dorsal midline and invade the field of the ablated homologous neuron [80]. The analysis of overlapping for the four classes of dendritic field revealed that dendritic arbors from neurons of the same class do not overlap, whereas dendritic arbors of different classes do overlap with each other [73]. This non-redundant coverage of the body wall by dendritic fields is referred to as tiling. In flamingo mutants, dendrites of md neurons overextend and invade the homologous contralateral dendritic field [80], suggesting a possible role of flamingo in inhibiting overlaps in dendritic fields of homologous class.

PNS dendrites are peculiar in the sense that they do not have specific pre-synaptic partners as would CNS neurons integrated in a network. These PNS neurons collect inputs generated by the external environment of the animal, and they do so by spreading over the body wall. It is still unclear to what extent intrinsic programming 
ensures how CNS neurons project their dendrites to the right target field and adopt the right conformation before local dialogue between pre- and post-synaptic partners establishes connections.

\section{Targeting}

Establishment of functional circuitry requires that each neuron of the network is able to find and connect with the right target among multiple similar targets. In a multilayered CNS, axons travel through layers and stop at the right layer. Then, in their target layer, the axons identify their appropriate post-synaptic partners. Here, we describe two model systems, although one in more depth than the other, that have given insight into the molecular mechanisms underlying neural targeting during development.

\section{Photoreceptors}

The eye of Drosophila is a good model to study the targeting of a specific layer in a three-dimensional system. It consists of 800 or so repeated simple units, or ommatidia, containing 8 different photoreceptor cells, R1-R8. R cells send their projections to different layers of the optic lobe. The photoreceptors R1-R6 project to the lamina between two layers of glial cells where they contact their post-synaptic partners to form structures called cartridges. The photoreceptors R7 and R8 navigate through the lamina without stopping and project deeper into the optic lobe to 2 different layers of the medulla, R7 synapsing deeper than R8. In the lamina the matching of photoreceptors with cartridges is not one ommatidium/one cartridge, but rather one point in the visual space/one cartridge [85]. The matching of the number of afferents to their targets is under the control of inductive signals from the $\mathrm{R}$ cells [86].

During their axon development, the $\mathrm{R}$ cells of each ommatidia project their axon to the optic lobe in a sequential manner that mimics their birth order. R8 enters the optic lobe first, while R1-R6 and finally R7 follow along the $\mathrm{R} 8$ tract. Having reached the optic lobe the $\mathrm{R}$ cell travels along the surface of the brain before turning medially to penetrate the optic lobe to seek its target layer.

To gain insight into this question, the ability to create mitotic mutant clones in a heterozygous animal using FLP/FRP system [34] has been refined to create homozygous mutant clones only in the eye, with high frequency and, furthermore, with elimination of the homozygous wild-type twin clone [87, 88]. Screening for mutations affecting the targeting of the $\mathrm{R}$ cells to the lamina or the medulla have allowed identification of several molecules involved in this process. These studies have shown that different molecules control the targeting to the different layers by the different $\mathrm{R}$ cells.

For example, the study of brakeless mutant clones has revealed its requirement in the targeting of lamina by $\mathrm{R} 1-$ R6 photoreceptors. In the absence of brakeless, R1-R6 axons are mistargeted to the medulla where they stop at the same level as R7 [89, 90]. This mistargeting can be suppressed when brakeless is resupplied to R1-R6 photoreceptor cells, showing its requirement for targeting to the lamina. However, ectopic expression of Brakeless in $\mathrm{R} 7$ and $\mathrm{R} 8$ is not sufficient to mistarget their axons to the lamina $[89,90]$. This suggests that other essential factors, whose activation is independent of brakeless, for the targeting of the lamina are expressed in R1-R6 photoreceptors and not in R7 and R8.

While no apparent change in cell-fate specification is noted in brakeless mutant clones [89, 90], runt, a transcription factor whose expression is restricted to $\mathrm{R} 7$ and $\mathrm{R} 8$, is found ectopically expressed in $\mathrm{R} 2$ and $\mathrm{R} 5$ in brakeless mutant clones [91]. Whereas ablation of runt from R7 and $\mathrm{R} 8$ does not produce a targeting defect, possibly due to redundancy with two other runt-related genes, its ectopic expression in R2 and R5 reproduces the mistargeting observed in brakeless mutants without changes in cell fate [91]. This suggests that brakeless controls the targeting of R2 and R5 by repressing the expression of runt. Since the other photoreceptors which usually target the lamina are also mistargeted in brakeless mutants, Brakeless might control targeting to the lamina by repressing other transcription factors yet to be identified and/or by activating specific transcription programs in these photoreceptors.

Besides the role of brakeless in R1-R6 layer targeting, it has been shown that R1-R6 axons lacking ptp69D, a receptor protein tyrosine phosphatase, are mistargeted to the medulla [87, 92]. Whereas ptp69D is cell-autonomously required for the targeting of R1-R6 termini, its misexpression in R7 and R8 does not retarget their axons to the lamina, indicating that ptp69D is required but not sufficient for lamina targeting [87, 92]. By analogy to its function in embryos $[93,94]$, ptp69D might receive a signal instructing the axons to defasciculate and take a path different from the $\mathrm{R} 8$ trajectory. In that case, defects would be expected also for R7 and, indeed, R7 fails to reach its target layer and stalls in the $\mathrm{R} 8$ layer [87]. 
A similar phenotype where R7 axons are apparently unable to extend over the R8 layer to reach their deeper target layer has also been found in mutants for another receptor protein tyrosine phosphatase (RPTP) called LAR $[95,96]$. Analysis of the developmental time course of R7s mutant for LAR has shown that they actually pass through the R8 layer to reach their correct, deeper layer before retracting to the $\mathrm{R} 8$ layer at later stages. Thus, LAR is not required for targeting R7 axons to their layer, but for maintaining their interactions with post-synaptic partners once they have reached them $[95,96]$. Genetic analysis has shown that increasing the amount of ptp69D in LAR mutant $\mathrm{R}$ cells does not compensate for the absence of LAR, but that overexpression of LAR in R cells can compensate for the lack of Ptp69D [95]. This, and also the use of chimeras between ptp69D and LAR, suggests that these proteins may be activating the same intracellular pathway to control R7 layer targeting. But LAR is also likely to possess unique and specific extracellular ligands [95]. The function of LAR in layer targeting seems to be restricted to R7, whereas Ptp69D controls targeting of R1-R6 photoreceptors and R7. This suggests a control of layer targeting with a combinatorial code of protein phosphatases, the specificity of R7 targeting being given by LAR and its specific ligand(s), as shown by the possibility to rescue the mistargeting of R7 axons depleted of ptp69D with LAR overexpression.

As with the embryonic CNS midline, the layered targeting of photoreceptors appears to use the same molecular tools to project and target correctly. As for midline crossing and selection of lateral pathways, the targeting of a layer by a neuron relies strongly on interactions with environment through receptors. In the eye system, while attraction and repulsion mechanisms have not been clearly identified, it is clear that neurons use extracellular signals to select their layers. It is not clear whether an attraction/repulsion mechanism is required for layer targeting, or establishing a 'stop or go' signal is sufficient. If attraction/repulsion signaling is translated into a sticking/sliding signaling, it is possible that the layer targeting is a combination of both type of signals.

Once the photoreceptor axons have reached their target layer, they are confronted by the choice of a specific target inside a layer to constitute a precise topographic map. This phase of neuron growth and targeting is almost the final step of networking, just before synaptogenesis. It involves a set of adhesion molecules. Injection of fluorescent dye into single ommatidia allows the visualization of the pattern of synaptogenesis. In wild-type optic lamina, R1-R6 axons enter their target layer as an ordered bun- dle. R1-R6 axons from each single ommatidium will then defasciculate, contact their target neurons arranged in columns and establish synapses with them, forming cartridges. $N$-Cadherin mutant photoreceptors R1-R6 present a correct targeting of their layer contrary to what is observed for R7 photoreceptors [97]. However, when depleted of N-cadherin, most of the R1-R6 photoreceptor axons do not defasciculate out of the bundle and do not select the correct synaptic partners [97]. Similar disruptions of the topographic map are observed for R8 photoreceptors [97]. This suggests that N-cadherin activity is required for the defasciculation of the axon termini.

Whereas the control of defasciculation seems to be assured by $\mathrm{N}$-cadherin function, flamingo, another member of the $N$-cadherin gene family, is thought likely to be involved in the selection of the synaptic partners by R1R6 and R8 photoreceptors [98, 99]. Flamingo mutant R1-R6 axons from each single ommatidium establish contact with different, randomly selected columns, forming cartridges that are under- or over-innervated, breaking the regular spacing of the termini [98]. Similarly, R8 axon termini do not defasciculate regularly, creating overlaps. In addition, some of the $\mathrm{R} 8$ axon termini retract to a more superficial layer, presumably due to a failure to stabilize contact with post-synaptic partners [98, 99]. These defects in the spacing of the axons are reminiscent of tiling defects observed in da neurons in the larval PNS. Flamingo being an homophilic cell-adhesion molecule, one possibility is that growth cones expressing Flamingo establish homophilic interactions triggering repulsion, which keeps each axon regularly spaced.

\section{Neuromuscular System}

Once the neuronal process has been successfully targeted to its synaptic partners, synaptogenesis occurs. This has been best studied at the level of genetics, development, physiology and plasticity using the neuromuscular junction between motoneurons and skeletal muscles in Drosophila embryos and larvae. Numerous reviews have covered the vast amount of knowledge generated by these studies [100-103]. The Drosophila neuromuscular junction still remains an essential and invaluable model for studying target recognition, synaptogenesis and synaptic plasticity from which we can learn about the final steps of the development of functional neuronetworks.

\section{Future Directions}

The different neuronal models presented in this review highlight the successive steps that a neuron achieves to form a functional neuronetwork. As shown here, the Dro- 
sophila nervous system provides a series of models for the analyses of neural development, from determination (not covered here) to differentiation.

The studies on guidance, branching and targeting suggest that the differentiation of axons and dendrites is regulated independently. Axon and dendrites from the same cell respond differently, independently to a similar environment, as shown by the guidance of axons and dendrites at the CNS midline. This suggests the existence of a regulatory system that can segregate axons from dendrites. Several non-exclusive hypotheses can be proposed for such regulatory systems. Since dendrites develop later than axon, timing could account for the independence observed between axon and dendrites. Alternatively, specific control of key molecule localization using different targeting sequences or different motors could generate differences between axons and dendrites. Finally neurons could use the targeting of alternative splice forms with different properties to different compartments to generate such asymmetry in response to signaling.

Neurons rely on extrinsic cues provided by their environment for their differentiation and integration into networks. This is illustrated at the CNS midline where neurons, through Robos and Frazzled receptors, receive Slit and Netrins signaling provided by midline glia to grow toward or away from the midline. This interaction with the environment is also found during axon and dendritic branching as well as during targeting and synaptogenesis, as shown by the involvement of several different celladhesion molecules in these processes. This suggests that at each time point in their development, neurons are capable of integrating information provided by the environment to their processes, whether they are axons, dendrites, or sister branches, and respond appropriately to these extrinsic cues.

During CNS midline guidance, the downregulation of Roundabout requires Commissureless associated to DNedd4, a ubiquitin ligase, which suggests a role for the polyubiquitined protein degradation system in axon guidance. Later in development, the polyubiquitinated protein degradation system as been involved in axon pruning during metamorphosis. Similarly, Rho family GTPases control growth, guidance, branching and targeting in different systems and in response to different stimuli. Either the use of the ubiquitinated protein degradation system or the use of Rho family GTPases at successive steps of development is capable of triggering different responses. This shows the versatility of several molecules or several mechanisms that are successfully reused. This suggests that neurons possess strong control systems which allow this versatility.
Identification of the molecular mechanisms that control the differentiation of neurons and their integration in a functional neuronetwork suggests that neurons are continually capable of integrating complex information provided by extrinsic cues and their genetic programming, through the use of similar pathways. Neurons are then able to respond appropriately to the vast amount of cues they received. This suggests the existence of multilayered control systems. Thus we are just at the beginning of understanding how exquisitely precise neuronetworks are established and that the molecules and molecular mechanisms already identified represent only the tip of the iceberg.

\section{Conclusion}

Invertebrate nervous systems have been perceived as small and relatively simple units. Analyses of the neuronetworks underlying behaviors, as well as the characterization of developmental mechanisms leading to their formation, illuminate the complexity of elaborating and using a functional neuronetwork. Drosophila provides a model integrating genetic developmental analyses and complex neuronetworks. It also stresses that constructing a functional neuronetwork, requires communication, adhesion and exchanges.

Thus, all you need is love [104] ... and good genetics! [105].

\section{Acknowledgements}

We thank the current members of the Chiba Laboratory for comments on the manuscript. The work in the laboratory is supported by grants from NIH/NINDS to A.C.
46

Neurosignals 2004;13:37-49
Furrer/Chiba 


\section{References}

1 Tessier-Lavigne M, Goodman CS: The molecular biology of axon guidance. Science 1996;274: 1123-1133.

2 Schmid A, Chiba A, Doe CQ: Clonal analysis of Drosophila embryonic neuroblasts: Neural cell types, axon projections and muscle targets. Development 1999;126:4653-4689.

3 Landgraf M, Bossing T, Technau GM, Bate M The origin, location, and projections of the embryonic abdominal motorneurons of Drosophila. J Neurosci 1997;17:9642-9655.

4 Seeger M, Tear G, Ferres-Marco D, Goodman CS: Mutations affecting growth cone guidance in Drosophila: Genes necessary for guidance toward or away from the midline. Neuron 1993;10:409-426.

5 Jacobs JR: The midline glia of Drosophila: A molecular genetic model for the developmental functions of glia. Prog Neurobiol 2000;62:475508 .

6 Harris R, Sabatelli LM, Seeger MA: Guidance cues at the Drosophila CNS midline: Identification and characterization of two Drosophila Netrin/UNC-6 homologs. Neuron 1996;17: 217-228.

7 Kolodziej PA, Timpe LC, Mitchell KJ, Fried SR, Goodman CS, Jan LY, Jan YN: Frazzled encodes a Drosophila member of the DCC immunoglobulin subfamily and is required for CNS and motor axon guidance. Cell 1996;87 197-204.

8 Mitchell KJ, Doyle JL, Serafini T, Kennedy TE, Tessier-Lavigne M, Goodman CS, Dickson BJ: Genetic analysis of Netrin genes in Drosophila: Netrins guide CNS commissural axons and peripheral motor axons. Neuron 1996;17: 203-215.

9 Keleman K, Dickson BJ: Short- and long-range repulsion by the Drosophila Unc5 netrin receptor. Neuron 2001;32:605-617.

10 Battye R, Stevens A, Jacobs JR: Axon repulsion from the midline of the Drosophila CNS requires slit function. Development 1999;126. 2475-2481.

11 Kidd T, Bland KS, Goodman CS: Slit is the midline repellent for the robo receptor in Drosophila. Cell 1999;96:785-794.

12 Simpson JH, Kidd T, Bland KS, Goodman CS Short-range and long-range guidance by slit and its Robo receptors. Robo and Robo 2 play distinct roles in midline guidance. Neuron 2000;28:753-766.

13 Rajagopalan S, Vivancos V, Nicolas E, Dickson BJ: Selecting a longitudinal pathway: Robo receptors specify the lateral position of axons in the Drosophila CNS. Cell 2000;103:10331045.

14 Rhee J, Mahfooz NS, Arregui C, Lilien J, Balsamo J, VanBerkum MF: Activation of the repulsive receptor Roundabout inhibits N-cadherinmediated cell adhesion. Nat Cell Biol 2002;4 798-805.
15 Kidd T, Brose K, Mitchell KJ, Fetter RD, Tessier-Lavigne M, Goodman CS, Tear G: Roundabout controls axon crossing of the CNS midline and defines a novel subfamily of evolutionarily conserved guidance receptors. Cell 1998;92:205-215.

16 Keleman K, Rajagopalan S, Cleppien D, Teis D, Paiha K, Huber LA, Technau GM, Dickson BJ: Comm sorts robo to control axon guidance at the Drosophila midline. Cell 2002;110:415427

17 Myat A, Henry P, McCabe V, Flintoft L, Rotin D, Tear G: Drosophila Nedd4, a ubiquitin ligase, is recruited by Commissureless to control cell surface levels of the roundabout receptor. Neuron 2002:35:447-459.

18 Georgiou M, Tear G: Commissureless is required both in commissural neurones and midline cells for axon guidance across the midline. Development 2002;129:2947-2956.

19 Brand AH, Perrimon N: Targeted gene expression as a means of altering cell fates and generating dominant phenotypes. Development 1993;118:401-415.

20 Cash S, Chiba A, Keshishian H: Alternate neuromuscular target selection following the loss of single muscle fibers in Drosophila. J Neurosci 1992:12:2051-2064.

21 Bossing T, Technau GM: The fate of the CNS midline progenitors in Drosophila as revealed by a new method for single cell labelling. Development 1994;120:1895-1906.

22 Wolf BD, Chiba A: Axon pathfinding proceeds normally despite disrupted growth cone decisions at CNS midline. Development 2000;127: 2001-2009.

23 Furrer MP, Kim S, Wolf B, Chiba A: Robo and Frazzled/DCC mediate dendritic guidance at the CNS midline. Nat Neurosci 2003;6:223230.

24 Stein E, Tessier-Lavigne M: Hierarchical organization of guidance receptors: Silencing of netrin attraction by slit through a Robo/DCC receptor complex. Science 2001;291:19281938.

25 Lin DM, Fetter RD, Kopczynski C, Grenningloh G, Goodman CS: Genetic analysis of Fasciclin II in Drosophila: Defasciculation, refasciculation, and altered fasciculation. Neuron 1994;13:1055-1069.

26 Prokop A, Uhler J, Roote J, Bate M: The kakapo mutation affects terminal arborization and central dendritic sprouting of Drosophila motorneurons. J Cell Biol 1998;143:1283-1294.

27 Zlatic M, Landgraf M, Bate M: Genetic specification of axonal arbors: Atonal regulates robo3 to position terminal branches in the Drosophila nervous system. Neuron 2003;37:41-51.

28 Simpson JH, Bland KS, Fetter RD, Goodman CS: Short-range and long-range guidance by Slit and its Robo receptors: A combinatorial code of Robo receptors controls lateral position. Cell 2000;103:1019-1032.

29 Rajagopalan S, Nicolas E, Vivancos V, Berger J, Dickson BJ: Crossing the midline: Roles and regulation of Robo receptors. Neuron 2000;28: 767-777.
30 Merritt DJ, Whitington PM: Central projections of sensory neurons in the Drosophila embryo correlate with sensory modality, soma position, and proneural gene function. J Neurosci 1995; 15:1755-1767.

31 Whitford KL, Marillat V, Stein E, Goodman CS, Tessier-Lavigne M, Chedotal A, Ghosh A Regulation of cortical dendrite development by Slit-Robo interactions. Neuron 2002;33:4761

32 Hausser M, Spruston N, Stuart GJ: Diversity and dynamics of dendritic signaling. Science 2000;290:739-744.

33 Koch C, Segev I: The role of single neurons in information processing. Nat Neurosci 2000 3(suppl):1171-1177.

34 Golic KG, Lindquist S: The FLP recombinase of yeast catalyzes site-specific recombination in the Drosophila genome. Cell 1989;59:499509.

35 Lee T, Luo L: Mosaic analysis with a repressible cell marker for studies of gene function in neuronal morphogenesis. Neuron 1999;22: 451-461.

36 Bastmeyer M, O’Leary DD: Dynamics of target recognition by interstitial axon branching along developing cortical axons. J Neurosci 1996;16: 1450-1459.

37 Matheson SF, Levine RB: Steroid hormone enhancement of neurite outgrowth in identified insect motor neurons involves specific effects on growth cone form and function. J Neurobiol 1999;38:27-45.

38 Yang MY, Armstrong JD, Vilinsky I, Strausfeld NJ, Kaiser K: Subdivision of the Drosophila mushroom bodies by enhancer-trap expression patterns. Neuron 1995;15:45-54.

39 Ito K, Suzuki K, Estes P, Ramaswami M, Yamamoto D, Strausfeld NJ: The organization of extrinsic neurons and their implications in the functional roles of the mushroom bodies in Drosophila melanogaster Meigen. Learn Mem 1998;5:52-77.

40 Strausfeld NJ, Hansen L, Li Y, Gomez RS, Ito $\mathrm{K}$ : Evolution, discovery, and interpretations of arthropod mushroom bodies. Learn Mem 1998;5:11-37.

41 Davis RL: Physiology and biochemistry of Drosophila learning mutants. Physiol Rev 1996;76: 299-317.

42 Heisenberg M: What do the mushroom bodies do for the insect brain? An introduction. Learn Mem 1998;5:1-10.

43 Ito K, Awano W, Suzuki K, Hiromi Y, Yamamoto D: The Drosophila mushroom body is a quadruple structure of clonal units each of which contains a virtually identical set of neurones and glial cells. Development 1997;124: 761-771.

44 Lee T, Lee A, Luo L: Development of the Drosophila mushroom bodies: Sequential generation of three distinct types of neurons from a neuroblast. Development 1999;126:40654076 
45 Crittenden JR, Skoulakis EM, Han KA, Kalderon D, Davis RL: Tripartite mushroom body architecture revealed by antigenic markers. Learn Mem 1998;5:38-51.

46 Noveen A, Daniel A, Hartenstein V: Early development of the Drosophila mushroom body: The roles of eyeless and dachshund. Development 2000;127:3475-3488.

47 Truman JW, Bate M: Spatial and temporal patterns of neurogenesis in the central nervous system of Drosophila melanogaster. Dev Biol 1988; 125:145-157.

48 Ito K, Hotta Y: Proliferation pattern of postembryonic neuroblasts in the brain of Drosophila melanogaster. Dev Biol 1992;149:134148.

49 Kurusu M, Awasaki T, Masuda-Nakagawa LM, Kawauchi H, Ito K, Furukubo-Tokunaga $\mathrm{K}$ : Embryonic and larval development of the Drosophila mushroom bodies: Concentric layer subdivisions and the role of fasciclin II. Development 2002;129:409-419.

50 Watts RJ, Hoopfer ED, Luo L: Axon pruning during Drosophila metamorphosis: Evidence for local degeneration and requirement of the ubiquitin-proteasome system. Neuron 2003; 38:871-885.

51 Wang J, Zugates CT, Liang IH, Lee CH, Lee T: Drosophila Dscam is required for divergent segregation of sister branches and suppresses ectopic bifurcation of axons. Neuron 2002;33. 559-571.

52 Schmucker D, Clemens JC, Shu H, Worby CA, Xiao J, Muda M, Dixon JE, Zipursky SL: Drosophila Dscam is an axon guidance receptor exhibiting extraordinary molecular diversity. Cell 2000;101:671-684.

53 Hummel T, Vasconcelos ML, Clemens JC, Fishilevich Y, Vosshall LB, Zipursky SL: Axonal targeting of olfactory receptor neurons in Drosophila is controlled by Dscam. Neuron 2003; 37:221-231.

54 Ng J, Nardine T, Harms M, Tzu J, Goldstein A, Sun Y, Dietzl G, Dickson BJ, Luo L: Rac GTPases control axon growth, guidance and branching. Nature 2002;416:442-447.

55 Truman JW: Metamorphosis of the central nervous system of Drosophila. J Neurobiol 1990; 21:1072-1084.

56 Tissot M, Stocker RF: Metamorphosis in Drosophila and other insects: The fate of neurons throughout the stages. Prog Neurobiol 2000;62: 89-111.

57 Bender M, Imam FB, Talbot WS, Ganetzky B, Hogness DS: Drosophila ecdysone receptor mutations reveal functional differences among receptor isoforms. Cell 1997;91:777-788.

58 Truman JW, Talbot WS, Fahrbach SE, Hogness DS: Ecdysone receptor expression in the CNS correlates with stage-specific responses to ecdysteroids during Drosophila and Manduca development. Development 1994;120:219_ 234.

59 Lee T, Marticke S, Sung C, Robinow S, Luo L: Cell-autonomous requirement of the USP/ EcR-B ecdysone receptor for mushroom body neuronal remodeling in Drosophila. Neuron 2000;28:807-818.
60 Brummel T, Abdollah S, Haerry TE, Shimell MJ, Merriam J, Raftery L, Wrana JL, O'Connor MB: The Drosophila activin receptor baboon signals through $\mathrm{dSmad} 2$ and controls cell proliferation but not patterning during larval development. Genes Dev 1999;13:98-111.

61 Newfeld SJ, Wisotzkey RG, Kumar S: Molecular evolution of a developmental pathway: Phylogenetic analyses of transforming growth factor-beta family ligands, receptors and Smad signal transducers. Genetics 1999;152:783795.

62 Chen Y, Riese MJ, Killinger MA, Hoffmann FM: A genetic screen for modifiers of Drosophila decapentaplegic signaling identifies mutations in punt, Mothers against dpp and the BMP-7 homologue, 60A. Development 1998; 125:1759-1768.

63 Aberle H, Haghighi AP, Fetter RD, McCabe BD, Magalhaes TR, Goodman CS: Wishful thinking encodes a BMP type II receptor that regulates synaptic growth in Drosophila. Neuron 2002;33:545-558.

64 Marques G, Bao H, Haerry TE, Shimell MJ, Duchek P, Zhang B, O'Connor MB: The Drosophila BMP type II receptor Wishful Thinking regulates neuromuscular synapse morphology and function. Neuron 2002;33:529-543.

65 Zheng X, Wang J, Haerry TE, Wu AY, Martin J, O'Connor MB, Lee CH, Lee T: TGF-beta signaling activates steroid hormone receptor expression during neuronal remodeling in the Drosophila brain. Cell 2003;112:303-315.

66 Das P, Inoue H, Baker JC, Beppu H, Kawabata M, Harland RM, Miyazono K, Padgett RW: Drosophila dSmad2 and Atr-I transmit activin/ TGFbeta signals. Genes Cells 1999;4:123134.

67 Jan YN, Jan LY: HLH proteins, fly neurogenesis, and vertebrate myogenesis. Cell 1993;75: $827-830$

68 Bodmer R, Jan YN: Morphological differentiation of the embryonic peripheral neurons in Drosophila. Rouxs Arch Dev Biol 1987;196: 69-77.

69 Bodmer R, Carretto R, Jan YN: Neurogenesis of the peripheral nervous system in Drosophila embryos: DNA replication patterns and cell lineages. Neuron 1989;3:21-32.

70 Brewster R, Bodmer R: Origin and specification of type II sensory neurons in Drosophila. Development 1995;121:2923-2936.

71 Bodmer R, Barbel S, Sheperd S, Jack JW, Jan LY, Jan YN: Transformation of sensory organs by mutations of the cut locus of $D$. melanogaster. Cell 1987;51:293-307.

72 Gao FB, Brenman JE, Jan LY, Jan YN: Genes regulating dendritic outgrowth, branching, and routing in Drosophila. Genes Dev 1999;13: 2549-2561.

73 Grueber WB, Jan LY, Jan YN: Tiling of the Drosophila epidermis by multidendritic sensory neurons. Development 2002;129:28672878.

74 Blochlinger K, Bodmer R, Jan LY, Jan YN: Patterns of expression of cut, a protein required for external sensory organ development in wild-type and cut mutant Drosophila embryos. Genes Dev 1990;4:1322-1331.
75 Grueber WB, Jan LY, Jan YN: Different levels of the homeodomain protein cut regulate distinct dendrite branching patterns of Drosophila multidendritic neurons. Cell 2003;112:805818.

76 Blochlinger K, Jan LY, Jan YN: Postembryonic patterns of expression of cut, a locus regulating sensory organ identity in Drosophila. Development 1993;117:441-450.

77 Moore AW, Jan LY, Jan YN: hamlet, a binary genetic switch between single- and multipledendrite neuron morphology. Science 2002; 297:1355-1358.

78 Brenman JE, Gao FB, Jan LY, Jan YN: Sequoia, a tramtrack-related zinc finger protein, functions as a pan-neural regulator for dendrite and axon morphogenesis in Drosophila. Dev Cell 2001;1:667-677.

79 Usui T, Shima Y, Shimada Y, Hirano S, Burgess RW, Schwarz TL, Takeichi M, Uemura T: Flamingo, a seven-pass transmembrane cadherin, regulates planar cell polarity under the control of Frizzled. Cell 1999;98:585-595.

80 Gao FB, Kohwi M, Brenman JE, Jan LY, Jan YN: Control of dendritic field formation in Drosophila: The roles of flamingo and competition between homologous neurons. Neuron 2000;28:91-101.

81 Sweeney NT, Li W, Gao FB: Genetic manipulation of single neurons in vivo reveals specific roles of flamingo in neuronal morphogenesis. Dev Biol 2002;247:76-88.

82 Reuter JE, Nardine TM, Penton A, Billuart P, Scott EK, Usui T, Uemura T, Luo L: A mosaic genetic screen for genes necessary for Drosophila mushroom body neuronal morphogenesis. Development 2003;130:1203-1213.

83 Li W, Gao FB: Actin filament-stabilizing protein tropomyosin regulates the size of dendritic fields. J Neurosci 2003;23:6171-6175.

84 Cooper JA: Actin dynamics: Tropomyosin provides stability. Curr Biol 2002;12:R523-R525.

85 Clandinin TR, Zipursky SL: Afferent growth cone interactions control synaptic specificity in the Drosophila visual system. Neuron 2000;28: 427-436.

86 Huang Z, Kunes S: Hedgehog, transmitted along retinal axons, triggers neurogenesis in the developing visual centers of the Drosophila brain. Cell 1996;86:411-422.

87 Newsome TP, Asling B, Dickson BJ: Analysis of Drosophila photoreceptor axon guidance in eye-specific mosaics. Development 2000;127: 851-860.

88 Stowers RS, Schwarz TL: A genetic method for generating Drosophila eyes composed exclusively of mitotic clones of a single genotype. Genetics 1999;152:1631-1639.

89 Senti K, Keleman K, Eisenhaber F, Dickson $\mathrm{BJ}$ : Brakeless is required for lamina targeting of R1-R6 axons in the Drosophila visual system. Development 2000;127:2291-2301.

90 Rao Y, Pang P, Ruan W, Gunning D, Zipursky $\mathrm{SL}$ : Brakeless is required for photoreceptor growth-cone targeting in Drosophila. Proc Nat Acad Sci USA 2000;97:5966-5971. 
91 Kaminker JS, Canon J, Salecker I, Banerjee U: Control of photoreceptor axon target choice by transcriptional repression of Runt. Nat Neurosci 2002;5:746-750

92 Garrity PA, Lee CH, Salecker I, Robertson HC, Desai CJ, Zinn K, Zipursky SL: Retinal axon target selection in Drosophila is regulated by a receptor protein tyrosine phosphatase. Neuron 1999;22:707-717.

93 Desai CJ, Gindhart JG Jr, Goldstein LS, Zinn $\mathrm{K}$ : Receptor tyrosine phosphatases are required for motor axon guidance in the Drosophila embryo. Cell 1996;84:599-609.

94 Desai CJ, Krueger NX, Saito H, Zinn K: Competition and cooperation among receptor tyrosine phosphatases control motoneuron growth cone guidance in Drosophila. Development 1997; 124:1941-1952.

95 Maurel-Zaffran C, Suzuki T, Gahmon G, Treisman JE, Dickson BJ: Cell-autonomous and -nonautonomous functions of LAR in R7 photoreceptor axon targeting. Neuron 2001;32: 225-235.
96 Clandinin TR, Lee CH, Herman T, Lee RC, Yang AY, Ovasapyan S, Zipursky SL: Drosophila LAR regulates $\mathrm{R} 1-\mathrm{R} 6$ and $\mathrm{R} 7$ target specificity in the visual system. Neuron 2001;32: 237-248.

97 Lee CH, Herman T, Clandinin TR, Lee R, Zipursky SL: N-Cadherin regulates target specificity in the Drosophila visual system. Neuron 2001;30:437-450.

98 Lee RC, Clandinin TR, Lee CH, Chen PL, Meinertzhagen IA, Zipursky SL: The protocadherin Flamingo is required for axon target selection in the Drosophila visual system. Nat Neurosci 2003;6:557-563.

99 Senti KA, Usui T, Boucke K, Greber U, Uemura T, Dickson BJ: Flamingo regulates r8 axonaxon and axon-target interactions in the Drosophila visual system. Curr Biol 2003;13:828832.
100 Keshishian H, Chiba A: Neuromuscular development in Drosophila: Insights from single neurons and single genes. Trends Neurosci 1993; 16:278-283.

101 Nose A, Van Vactor D, Auld V, Goodman CS: Development of neuromuscular specificity in Drosophila. Cold Spring Harb Symp Quant Biol 1992;57:441-449.

102 Chiba A: Early development of the Drosophila neuromuscular junction: A model for studying neuronal networks in development. Int Rev Neurobiol 1999;43:1-24.

103 Gramates LS, Budnik V: Assembly and maturation of the Drosophila larval neuromuscular junction. Int Rev Neurobiol 1999;43:93117.

104 Lennon J, McCartney P: All you need is love. Parlophone 1967;R5620.

105 Mendel G: Versuche über Pflanzen-Hybriden. Verhandlungen des naturforschenden Vereins, 1865 